\title{
Tolerability, safety and adherence to treatment with insulin detemir injection in the treatment of type 2 diabetes
}

\author{
Athena Philis-Tsimikas \\ Scripps Whittier Diabetes Institute, \\ La Jolla, CA, USA
}

\begin{abstract}
The progressive nature of type 2 diabetes poses challenges in the clinic: treatment must be continually reviewed and adjusted in response to the patient's changing pathophysiology. Ultimately, insulin replacement therapy will be necessary as the physiological insulin response is compromised. The modern basal insulin analog insulin detemir has been the subject of several clinical trials and observational studies in type 2 diabetes. Compared with NPH insulin, insulin detemir offers an improved balance between achieving current glycemic targets with acceptable tolerability. Insulin detemir also has a unique weight-sparing effect which is associated with body mass index, and this may be a particular advantage to obese patients with type 2 diabetes. This review summarizes data from key clinical studies of insulin detemir, and also provides insights from observational studies.
\end{abstract}

Keywords: type 2 diabetes mellitus, insulin detemir, modern analog, basal insulin

\section{Introduction}

At least 246 million people worldwide (age group 20-79 years) have diabetes (IDF 2006). For type 2 diabetes (the more common form of the disease) sedentary lifestyles, poor diets and aging populations are thought to contribute to the growing epidemic. In particular, the number of people with obesity is escalating, which poses a major risk factor for the onset type 2 diabetes (WHO 2008).

Type 2 diabetes is a metabolic disorder characterized by relative insulin deficiency resulting from a reduced sensitivity of tissues to insulin and impairment of insulin secretion from pancreatic beta-cells. This leads to chronic hyperglycemia with disturbances of carbohydrate and other nutrient metabolism, all of which increase the risk of health complications in the future (UKPDS 33 1998; UKPDS 35 2000). Complications such as cardiovascular disease account for 30\%-50\% of mortality amongst people with diabetes, and diabetic neuropathy and retinopathy are frequent morbidities in this patient group (UKPDS 33 1998; UKPDS 35 2000). Modern therapies aim to achieve levels of glycemic control close to those found in health, in order to reduce the risk of these complications.

Initially, treatment for type 2 diabetes relies on diet and lifestyle changes; metformin can be added quickly if there is no preliminary response. As the disease progresses, oral antidiabetic drugs (OADs) are administered, first as monotherapy, and later, as the condition progresses, as combination therapy using two or three OADs with additive or synergistic potential. However, many type 2 diabetes patients will still require insulin to maintain good glycemic control (Figure 1). Some authors have suggested initiating insulin early in the treatment paradigm (Nathan et al 2006) in order to maintain good glycemic control $\left(\mathrm{HbA}_{1 \mathrm{c}}<7 \%\right)$ and also to preserve beta-cell function, which is progressively diminished by over-exposure to hyperglycemia (Buchanan 2003). 


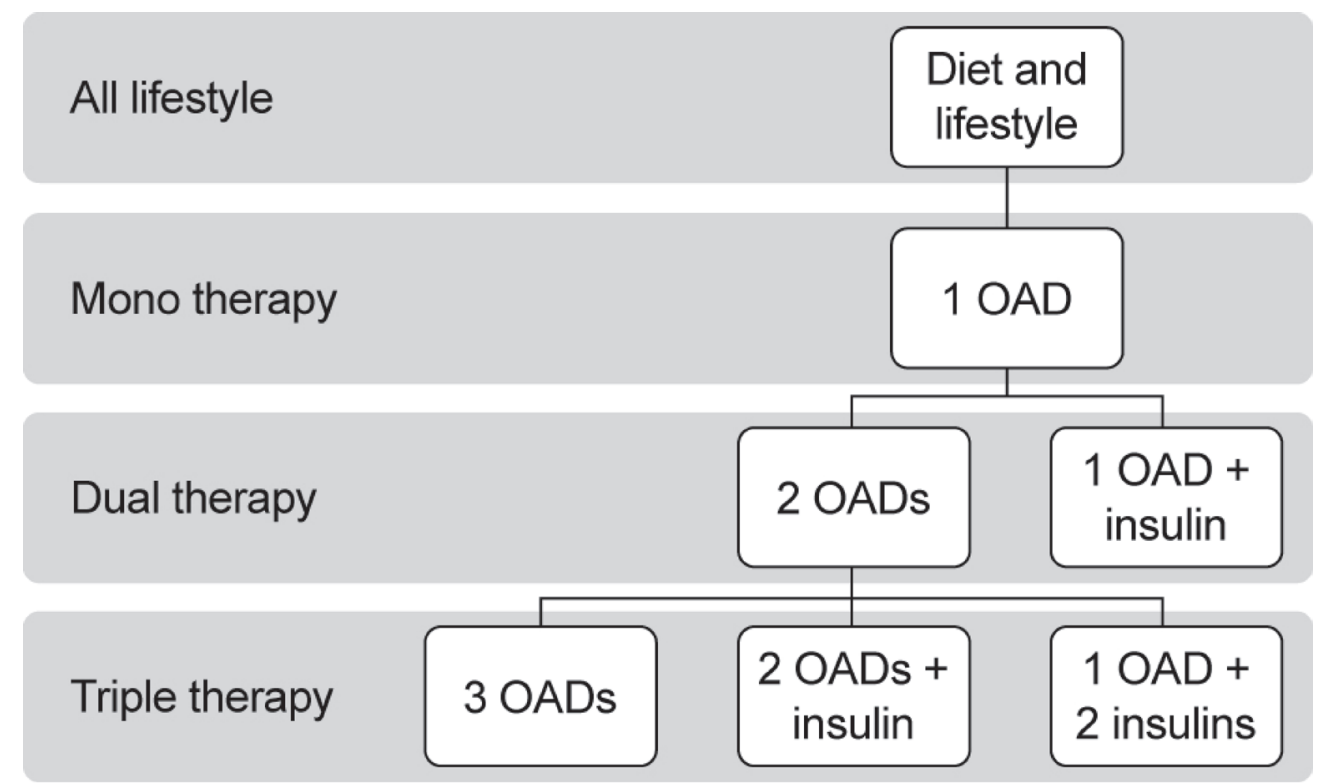

Figure I The current treatment cascade in type 2 diabetes.

\section{Barriers to the initiation of insulin therapy}

Despite the demonstrated benefits of initiating insulin therapy, there is often considerable resistance from both patients and physicians. Patients tend to feel apprehensive about injections, as they are perceived by many to be invasive and painful (Korytkowski 2002). This misconception may, in part, have been fostered by past experiences with older delivery systems such as syringes and vials.

Patients may also be anxious about hypoglycemia and weight gain, which are well recognized adverse effects associated with insulin therapy. These apprehensions are likely to negatively influence the prescribing attitudes of physicians towards insulin regimens (Korytkowski 2002; Home et al 2003; Davies 2004). This issue is highlighted by the Diabetes Attitudes Wishes and Needs (DAWN) study, a survey of health care professionals (nurses $=1,109$; physicians $=2,681)$ and insulin-naïve people with diabetes $(2,061)$ in 13 countries. The DAWN study reported that many health care providers, especially those in the USA, are inclined to delay initiation of insulin therapy as a result of concerns over patient adherence to an insulin-based regimen (Peyrot et al 2005).

Attitude-related barriers to insulin use are commonly referred to as 'psychological insulin resistance', which not only delays insulin initiation when blood glucose control worsens, but can also restrict insulin intensification, a modification required as the disease state progresses and beta-cell function deteriorates. Failure to initiate or intensify insulin in a timely fashion can have serious consequences, as even brief periods of poor glycemic control in individuals with type 2 diabetes ultimately increase the risk of micro- and macrovascular complications (UKPDS 35 2000).

\section{Insulin detemir: designed to support patient acceptance of basal insulin}

For many years, insulin therapy in type 2 diabetes has made use of older basal insulins such as neutral protamine Hagedorn (NPH) insulin. There are, however, important disadvantages with NPH insulin, in particular a short duration of action (less than 24 hours) (Lepore et al 2000), large variability in the glucose-lowering effect from injection to injection, and a pronounced peak activity in effect (Heise et al 2004). Within-subject variability of the pharmacodynamic effect may affect the extent to which blood glucose levels fluctuate in individual patients (Heise et al 2004). Superimposed on a peak effect around 4 hours after dosing followed by a decline (Heise and Pieber 2007), this variability translates into a major contributing factor to nocturnal hypoglycemia.

Insulin analogs have been engineered by adapting the human insulin molecular structure, with the aim of providing a more physiological insulin profile than exogenous human insulin in patients with diabetes. In particular, the amino acid sequence and physicochemical properties have been modified with an aim to overcome the pharmacokinetic shortcomings of exogenous human insulins. This, in turn, is 
intended to improve the balance between glycemic control and tolerability (Gough 2007; Heller et al 2007).

Unlike NPH insulin, insulin detemir's prolonged absorption profile is not achieved by the resuspension of crystals with subsequent dissolution in the subcutaneous tissue; neither, unlike insulin glargine, does it rely on the dissolution of microprecipitates formed after injection (Klein et al 2007). Instead, the prolonged absorption of insulin detemir is established by its self-association into stable hexamers, and the apparent ability of these to aggregate to form dihexamers when in high concentrations, such as in the subcutaneous injection site (Havelund et al 2004). Furthermore, insulin detemir has an acylated fatty acid chain that enables reversible binding to albumin at the injection depot. In the circulation, albumin binding also buffers the effect of changes in the absorption rate from the injection site, thereby contributing to reduced pharmacodynamic variability (Havelund et al 2004; Kurtzhals 2004). As a result, insulin detemir has a long and relatively flat time-action profile, which is consistent from one injection to another in comparison with NPH insulin and insulin glargine (Heise et al 2004; Klein et al 2007). This has recently been demonstrated in a cohort of patients with type 1 diabetes who were randomized to receive injections of insulin detemir or insulin glargine (Bock et al 2008). The authors concluded that an action profile of approximately 24 hours in the majority of patients made both insulins suitable for once-daily dosing, and a lower variation between injections was observed for patients randomized to insulin detemir (Bock et al 2008).

The pharmacodynamic properties of insulin detemir have been investigated in a series of glycemic clamp studies. Heise et al (2004) reported greater consistency in the glucose infusion rate time curves in patients with type 1 diabetes administered insulin detemir in a repeat clamp study, and this was reflected in the lower coefficients of variability in numerous pharmacodynamic endpoints. Significantly lower within-subject variability was demonstrated (as defined by coefficient of variation of glucose infusion rate, area under curve) with insulin detemir compared with NPH insulin and insulin glargine: $27 \%$ vs $34 \%$ vs $46 \%$, respectively (Heise et al 2004). Klein et al (2007) reported similar findings in patients with type 2 diabetes as within-subject variability was found to be significantly lower for the albumin-bound insulin analogs insulin detemir and an experimental acylated analog, NN344, than insulin glargine (Klein et al 2007). This suggests the lower within-subject variability observed with insulin detemir may be an inherent property of its ability to bind to albumin. (Heise and Pieber 2007; Klein et al 2007).

\section{Safety profile of insulin detemir}

Molecular alteration of human insulin to form insulin analogs can compromise binding properties, and also the metabolic and mitogenic potencies associated with endogenous human insulin, since this peptide hormone also has some affinity for the insulin-like growth factor I (IGF-I) receptor, which is associated with stimulation of mitosis. In vitro studies have demonstrated that the mitogenic potency of insulin analogs mostly correlates with their relative IGF-I receptor binding affinities and/or their rate of dissociation from the insulin receptor; analogs with high affinity for the IGF-I receptor and/or a low dissociation rate from the insulin receptor have increased mitogenic potency. Lessons learned from the toxicological implications of the insulin analog, B10Asp, an analog whose mitogenic potency was high, have informed the development of subsequent insulin analogs (Kurtzhals et al 2000). Insulin detemir has been shown to have a low mitogenic potential and a low IGF-I receptor affinity compared with human insulin (Kurtzhals et al 2000).

Albumin-binding has also been researched to confirm safety; no drug interactions involving other albumin-binding drugs have been identified, while the number of fatty acid binding sites available in circulating albumin exceed the number required for a therapeutic dose of insulin detemir (with a molar concentration in serum of only 1:50,000 to that of albumin) (Home and Kurtzhals 2006).

\section{Advantages of insulin detemir in the clinical arena}

Multicenter, open-label, parallel, randomized clinical phase III studies of 22-104 weeks in duration were conducted to compare the effect of insulin detemir, NPH insulin and insulin glargine on glycemic control, hypoglycemia and weight.

\section{Improving glycemic control and reducing hypoglycemic risk}

Randomized controlled trials have established insulin detemir to be as effective as NPH insulin (in both basal plus OAD therapy and basal plus bolus therapy) and insulin glargine (in basal plus OAD therapy) in improving glycemic control in type 2 diabetes (Rašlová et al 2004; Haak et al 2005; Hermansen et al 2006; Philis-Tsimikas et al 2006; Rosenstock et al 2008). Trials using aggressive 'treat-totarget' dose titration algorithms have found that the addition of insulin detemir to oral drug therapy in patients failing on OADs alone can reduce mean $\mathrm{HbA}_{1 \mathrm{c}}$ values in people with type 2 diabetes by approximately $1.5 \%$ (Hermansen et al 2006; Philis-Tsimikas et al 2006; Rosenstock et al 2008). 
Thus, if baseline $\mathrm{HbA}_{1 \mathrm{c}}$ is not already elevated above $8.5 \%$, the achievement of guideline targets can be expected in many individuals.

Hermansen et al (2006) reported a treat-to-target study comparing twice-daily insulin detemir with twice-daily NPH insulin in basal insulin plus OAD regimens.

In this 26-week, parallel-group, multicenter trial, 475 patients were randomized to treatment with insulin detemir or NPH insulin, adjusted to reach pre-breakfast and pre-dinner plasma glucose targets of $\leq 6 \mathrm{mmol} / \mathrm{L}$.

Patients from each treatment arm experienced similar reductions and improvements in $\mathrm{HbA}_{1 \mathrm{c}}$; this corresponded to a final mean $\mathrm{HbA}_{1 \mathrm{c}}$ of $6.8 \%$ with insulin detemir and $6.6 \%$ with NPH insulin, with a large proportion of all patients (70\%) reaching their target $\mathrm{HbA}_{1 \mathrm{c}}$ of $\leq 7.0 \%$. Moreover, the number of patients reaching this target without hypoglycemia was significantly higher with insulin detemir (34\%) than NPH insulin $(25 \% ; \mathrm{p}=0.008)$. The relative risk (RR) of overall and nocturnal hypoglycemia was lower with insulin detemir than NPH insulin (47\% and 55\% respectively, both $\mathrm{p}<0.01$ ), at equivalent levels of glycemic control (Hermansen et al 2006).

These findings were supported by a further study comparing insulin detemir with NPH insulin, in which we also considered the timing of the dose in the protocol, ie, a comparison of a pre-breakfast and evening dose of once-daily insulin detemir. Evening administration of insulin detemir was associated with a $65 \%$ risk reduction in confirmed nocturnal episodes versus evening NPH insulin $(p<0.05)$ at equivalent $\mathrm{HbA}_{1 \mathrm{c}}$, with the incidence of nocturnal hypoglycemia associated with morning detemir lower still (87\%, $\mathrm{p}<0.001$ ) (Philis-Tsimikas et al 2006). No statistically significant differences were obtained between AM and PM insulin detemir arms. Overall (24 hour) hypoglycemia was reduced by over $50 \%$ with evening insulin detemir compared with NPH insulin (Philis-Tsimikas et al 2006), suggesting a greater tolerability for insulin detemir at equivalent levels of glycemic control.

Rosenstock et al (2008) compared insulin detemir with insulin glargine in a parallel-group study in 582 insulin-naïve adults with type 2 diabetes. Baseline $\mathrm{HbA}_{1 \mathrm{c}}$ was reduced by both insulins from $8.6 \%$ to $7.2 \%$ for insulin detemir and to $7.1 \%$ for insulin glargine $(\mathrm{p}=\mathrm{ns})$. Incidence of overall hypoglycemia was low (5.8 vs 6.2 episodes per patient year for insulin detemir and glargine respectively, $\mathrm{p}=\mathrm{ns}$ ), with incidence of nocturnal hypoglycemia of 1.3 episodes per patient year for both, and major hypoglycemia too rare for statistical analyses to be performed (Rosenstock et al 2008).
A post-hoc analysis of three trials (Rašlová et al 2004; Haak et al 2005; Hermansen et al 2006), which were 22-26 weeks in duration, demonstrated the same reduced risk in hypoglycemia in the elderly (aged $\geq 65$ years) as in the younger age groups (aged 18-64 years), comparing insulin detemir with NPH insulin (Garber et al 2007). A risk reduction of $41 \%$ for all hypoglycemic episodes was observed with insulin detemir, which was statistically significant $(R R=0.59, p=0.002$, for older persons; $R R=0.75, p=0.02$, for younger persons) (Garber et al 2007). This risk reduction is particularly important, as it may encourage elderly patients to adhere to treatment intensification, as and when required.

Two studies have evaluated insulin detemir when used as part of a basal-bolus regimen in type 2 diabetes (Haak et al 2005; Rašlová et al 2004), and compared with NPH insulin. Both found that equivalent levels of glycemic control were achieved between study insulins, and a similar safety profile was observed. However, in both studies, two additional benefits were seen in the insulin detemir arm: reduced weight gain, and a significantly lower variation in fasting blood glucose (FBG) (Haak et al 2005; Rašlová et al 2004).

\section{Reducing variability}

Consistent with data from glycemic clamp studies, insulin detemir has been shown in clinical studies of patients with type 2 diabetes on basal-bolus therapy to reduce withinpatient variability in plasma glucose levels compared with $\mathrm{NPH}$ insulin (Table 1). Reductions in variability were reported in clinical studies involving basal-bolus regimens with insulin detemir, yet this was not always the case for the basal plus OAD studies (Philis-Tsimikas et al 2006). This inconsistency may be explained by the fact that patients treated with basal and OAD therapy are likely to have a

Table I Within-subject variability in type 2 diabetes patients treated with insulin detemir in comparison with NPH insulin

\begin{tabular}{|c|c|c|c|}
\hline \multirow[b]{2}{*}{ Study } & \multicolumn{3}{|c|}{$\begin{array}{l}\text { Day-to-day variation in self-monitored blood } \\
\text { glucose levels }\end{array}$} \\
\hline & Insulin detemir & NPH insulin & p-value \\
\hline Haak et al 2005 & 1.3 & 1.4 & 0.02 \\
\hline Rašlová et al 2004 & 1.2 & 1.5 & $<0.001$ \\
\hline $\begin{array}{l}\text { Hermansen } \\
\text { et al } 2006^{a}\end{array}$ & 1.3 & $\mathrm{I} .4$ & $<0.001$ \\
\hline $\begin{array}{l}\text { Philis-Tsimikas } \\
\text { et al } 2006\end{array}$ & $\mathrm{~N} / \mathrm{A}$ & $\mathrm{N} / \mathrm{A}$ & $\begin{array}{l}\text { No significant } \\
\text { differences } \\
\text { observed }\end{array}$ \\
\hline
\end{tabular}

aDetermined using pooled pre-breakfast and pre-dinner values. 
greater preservation of endogenous insulin response than those treated with basal-bolus, and an ability to regulate endogenous insulin secretion may mask between-treatment differences in the blood glucose-lowering effects of the study insulins.

The clinical implications of reduced blood glucose variability are, as yet, uncertain; one group found that the coefficient of variation of fasting plasma glucose (FPG) was an independent predictor of mortality (both all-cause and, in particular, cardiovascular mortality) (Muggeo et al 2000). However, the relationship between glucose fluctuations and macrovascular complications remains controversial, and is currently under debate (Kilpatrick et al 2006; Hirsch and Brownlee 2007; Monnier et al 2007). Recent research suggests that the reduced variability in the metabolic effect of insulin detemir is strongly associated with a reduced incidence of nocturnal hypoglycemia (Niskanen et al 2008), consistent with results seen in controlled clinical trials.

\section{Tolerability}

Notwithstanding the reduced incidence of hypoglycemia and the lower mean weight gain, in clinical trials of patients with type 2 diabetes, insulin detemir has been shown to be well tolerated, with few adverse events reported and a tolerability profile similar to other insulins (Rašlová et al 2004; Haak et al 2005; Hermansen et al 2006; Philis-Tsimikas et al 2006; Rosenstock et al 2008).

\section{Reducing the risk of weight gain}

Concern over the potential for weight gain can act as a significant barrier to insulin initiation in patients with type 2 diabetes (Korytkowski 2002), and can also deter patient adherence from intensive therapy required to optimize glycemic control (Polonsky et al 2005). Insulin detemir may help patients reach glycemic targets with significantly less weight gain than other insulins (Russell-Jones and Khan 2007).

At equivalent levels of glycemic control, insulin detemir has been found to be associated with significantly less weight gain than NPH insulin in a number of clinical trials both in basal-OAD regimens (Hermansen et al 2006; PhilisTsimikas et al 2006) and when used in basal-bolus therapy (Rašlová et al 2004; Haak et al 2005) (Figure 2). Insulin detemir also shows lower weight gain when compared with insulin glargine; a 1 year study in patients on a basal + OAD regimen showed that weight gain in insulin detemir-treated patients was significantly lower when compared with insulin glargine-treated patients ( $3.0 \mathrm{vs} 3.5 \mathrm{~kg}, \mathrm{p}<0.01)$, and patients completing the study on once-daily insulin detemir gained the least weight (2.25 kg) (Rosenstock et al 2008).

The reduced weight gain associated with insulin detemir compared with other basal insulins appears to have a correlation with body mass index (BMI): the two clinical trials comparing insulin detemir with NPH insulin as part of basal + OAD therapy both found that, with increasing baseline BMI, patients gained less weight with insulin

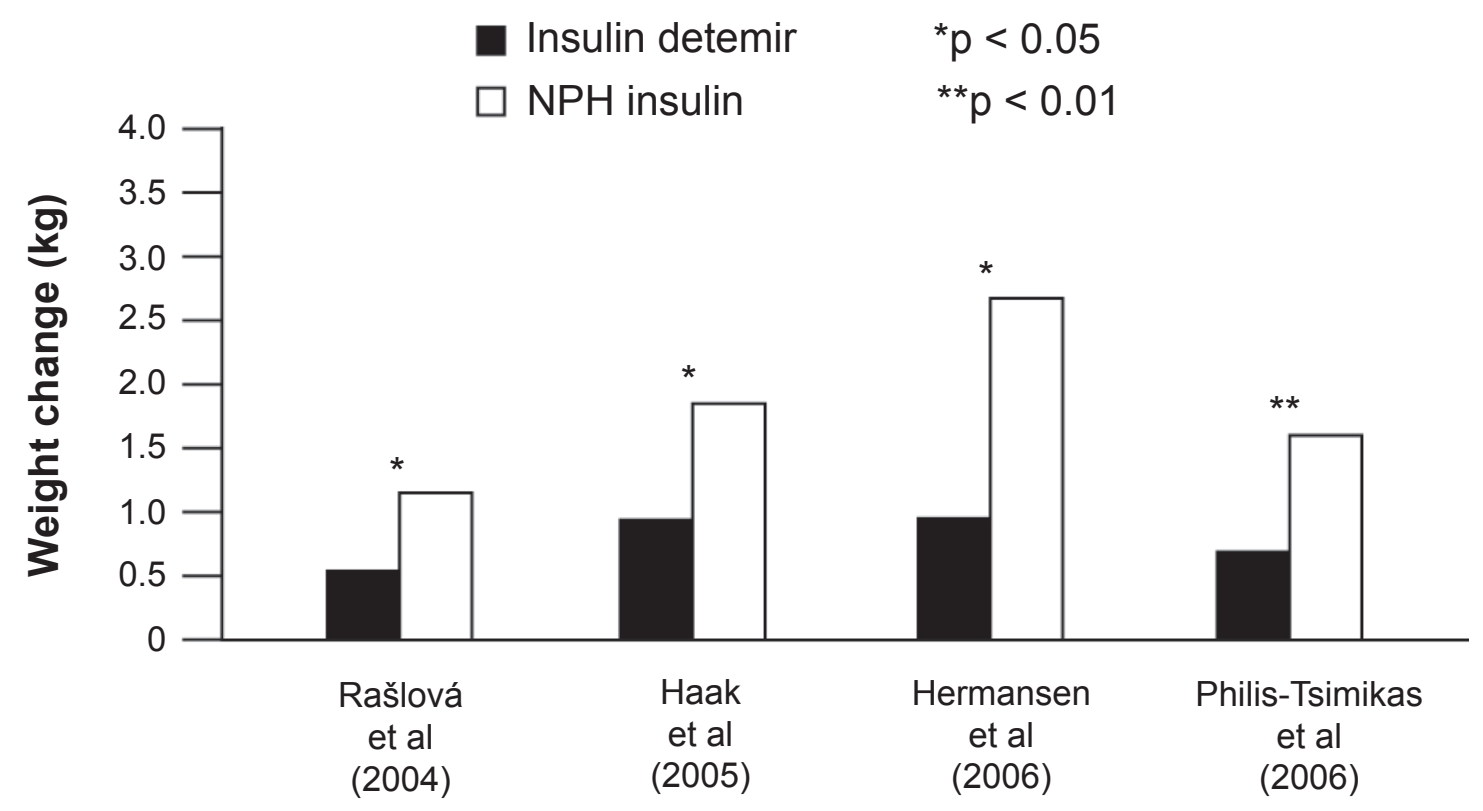

Figure 2 Change in weight reported in clinical trials comparing insulin detemir and NPH insulin in type 2 diabetes patients. Derived from data from Rašlová et al (2004); Haak et al (2005); Hermansen et al (2006); Philis-Tsimikas et al (2006). 
detemir (Hermansen et al 2006; Philis-Tsimikas et al 2006) (Figure 3); in both studies, this relationship was not found for NPH insulin ( $p=N S)$. Thus, insulin detemir may offer a weight advantage over NPH insulin, especially in overweight or obese people with type 2 diabetes initiating insulin therapy (Hermansen et al 2006; Philis-Tsimikas et al 2006).

\section{Phase IV studies}

Randomized controlled trials have played an important role in assessing the safety and efficacy of insulin detemir. However, it is also important to assess the effectiveness of insulin detemir in a real life setting. The PREDICTIVE ${ }^{\mathrm{TM}}$ study
(Predictable Results and Experience in Diabetes through Intensification and Control to Target: An International Variability Evaluation), is a large, open-label, non-randomized, non-interventional study, carried out in a diverse population primarily to evaluate the safety and efficacy of insulin detemir as part of routine clinical care (Lüddeke et al 2007). This multinational study has recruited, so far, as many as 40,000 patients, who have been followed for 12, 26 or 52 weeks, depending on country.

Data recorded for patients participating in PREDIC$\mathrm{TIVE}^{\mathrm{TM}}$ for 12 weeks have been recently published (Dornhorst et al 2007; Dornhorst et al 2008a; Dornhorst et al 2008b).
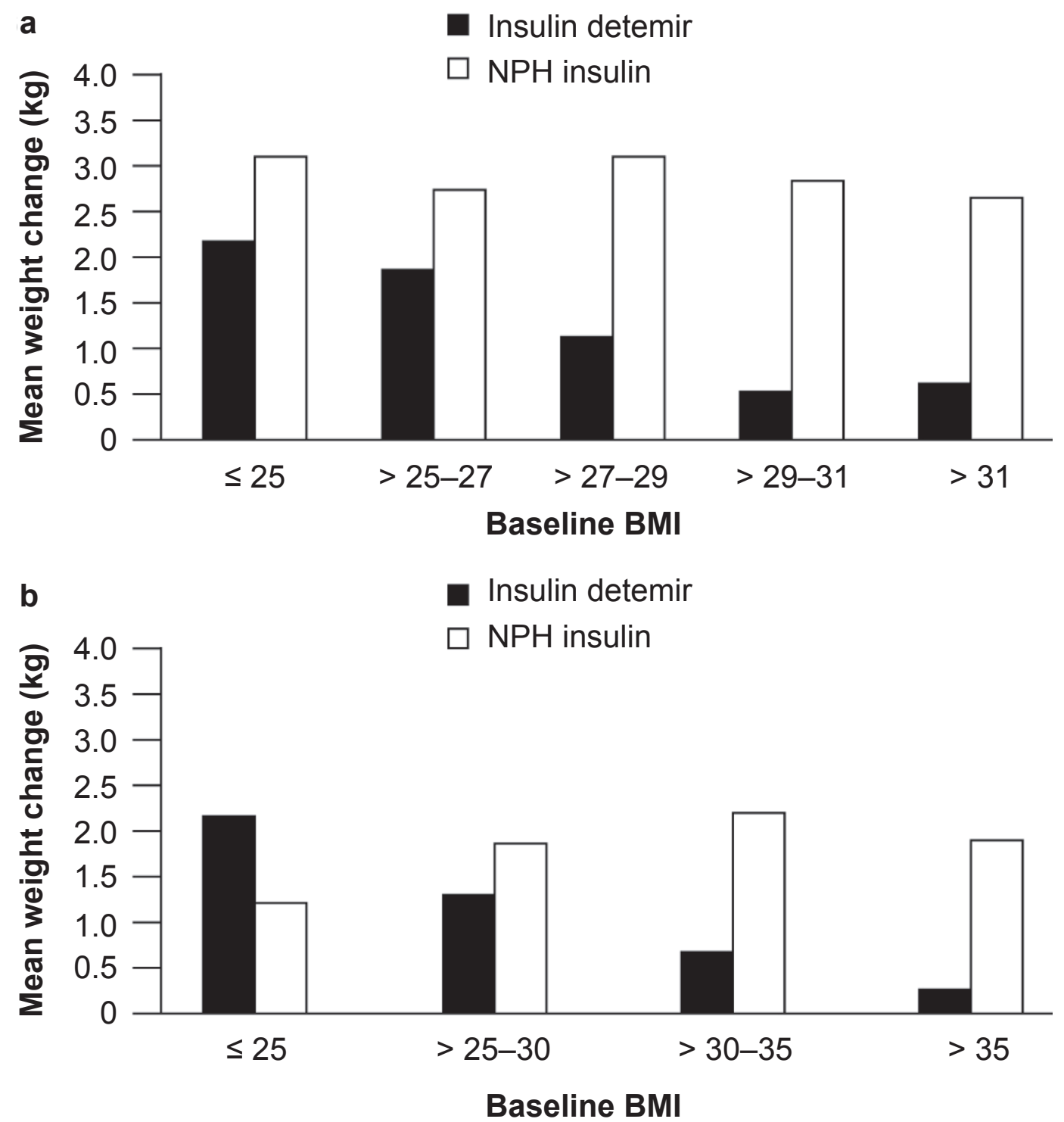

Figure 3 a) Weight change stratified by body mass index (BMI) in type 2 diabetes patients. Reprinted with permission from Hermansen K, Davies M, Derezinski T, et al 2006. A 26-week, randomized, parallel, treat-to-target trial comparing insulin detemir with NPH insulin as add-on therapy to oral glucose-lowering drugs in insulin-naïve people with type 2 diabetes. Diabetes Care, 29:1269-74. Copyright (C) 2006 American Diabetes Association. b) Weight change stratified by BMI in type 2 diabetes patients. Derived from data from Philis-Tsimikas et al (2006). 
The PREDICTIVE ${ }^{\mathrm{TM}}$ study included a large cohort of patients who were insulin-naïve prior to starting a regimen with insulin detemir (Dornhorst et al 2008a). Glycemic control improved significantly; mean $\mathrm{HbA}_{1 \mathrm{c}}$ was reduced from $8.9 \%$ to $7.6 \%(-1.3 \% ; \mathrm{p}<0.0001)$ and mean fasting glucose and within-patient fasting glucose variability was reduced by -3.7 and $-0.5 \mathrm{mmol} / \mathrm{L}$, respectively ( $p<0.0001)$. This was achieved in association with a low risk of hypoglycemia; the incidence of total, major and nocturnal episodes was low, with a slight reduction in incidence (baseline frequency:endpoint frequency): -0.3 (1.4-1.2), -0.1 (0.1-0.0), $-0.1(0.4-0.3)$ episodes per patient year, for total, major and nocturnal hypoglycemia, respectively. A statistically significant decrease in mean body weight $(-0.7 \mathrm{~kg} ; \mathrm{p}<0.0001)$ was also reported in these previously insulin-naïve patients. As observed in clinical trials, mean weight change $(\mathrm{kg})$ had a statistically significant inverse relationship with BMI, in which patients with the highest BMI $\left(\mathrm{BMI} \geq 31 \mathrm{~kg} / \mathrm{m}^{2}\right)$ experienced the greatest reduction in weight $(-1.51 \mathrm{~kg})$ (Dornhorst et al 2008a).

Patients switching from their previous basal insulin to insulin detemir experienced improvements in glycemic control: in the previously NPH insulin-treated group, mean $\mathrm{HbA}_{1 \mathrm{c}}$ was reduced by $-0.2 \%(\mathrm{p}<0.05)$ and by $-0.6 \%$ $(\mathrm{p}<0.0001)$ among patients switching from insulin glargine. The improvements in glycemic control were accompanied by significant reductions in hypoglycemia. Patients switching from NPH insulin and insulin glargine to insulin detemir had significant decreases in the incidence of total hypoglycemia from 11.7 to 3.0 episodes/patient-year $(p<0.001)$ and from 4.3 to 0.8 episodes/patient-year $(\mathrm{p}<0.01)$, respectively. Nocturnal hypoglycemia was also significantly reduced by $81 \%$ in patients switching from NPH insulin and by $75 \%$ in patients switching from insulin glargine (Figure 4). Switching to insulin detemir also resulted in a weight-sparing effect as mean body weight was reduced by $0.7 \mathrm{~kg}$ in NPH insulin switchers $(\mathrm{p}<0.01)$ and by $0.5 \mathrm{~kg}$ in insulin glargine switchers $(\mathrm{p}<0.05)$ (Dornhorst et al 2008b).

\section{Insulin detemir in a primary care setting}

Insulin initiation and treatment is increasingly managed in a primary care setting. The PREDICTIVE ${ }^{\mathrm{TM}} 303$ trial was a prospective trial of insulin detemir carried out predominantly in primary care practices in the USA (Meneghini et al 2007; Selam et al 2008). It compared a simplified patient-adjusted dosing algorithm (303) with a standard of care physiciandriven adjustment in individuals with type 2 diabetes.
All patients in the study were switched to, or started on, once-daily insulin detemir as their basal insulin replacement. Patients were randomized and the starting doses were $0.32 \mathrm{U} / \mathrm{kg}$, and $0.34 \mathrm{U} / \mathrm{kg}$ for the 303 and standard of care groups, respectively. The 303 algorithm group was instructed to use a simple algorithm to adjust their insulin detemir dose every 3 days based on three self-measured FBG values:

- Patients reduced their insulin dose by 3 units if FBG $<80 \mathrm{mg} / \mathrm{dL}(<4.4 \mathrm{mmol} / \mathrm{L})$;

- Patients made no change to their current insulin dose if FBG was between 80-110 mg/dL (4.4-6.1 mmol/L);

- Patients increased their insulin dose by 3 units if $\mathrm{FBG}>110 \mathrm{mg} / \mathrm{dL}(>6.1 \mathrm{mmol} / \mathrm{L})$.

Insulin adjustments in the standard of care group were made by the physician. A significant difference in reduction in $\mathrm{HbA}_{1 \mathrm{c}}$ was observed in favor of the 303 algorithm group ( $\mathrm{p}=0.016$ ), although the reductions from baseline (8.5\% for both groups) to endpoint ( 7.9 vs $8.0 \%)$ were of clinically similar magnitude. Despite similarities in $\mathrm{HbA}_{1 \mathrm{c}}$ reductions, the mean FPG reduction was significantly greater in the 303 algorithm group than the standard of care group; $1.8 \mathrm{mmol} / \mathrm{L}$ vs $1.2 \mathrm{mmol} / \mathrm{L}$, respectively ( $<<0.0001)$. Overall hypoglycemia was reduced in both groups by 2.61 from 9.53 events/patient-year and by 4.58 from 9.05 events/patient-year in the 303 algorithm and the standard of care group, respectively. Both patient groups had no significant weight gain after 26 weeks of treatment. Interestingly, an analysis of the entire PREDICTIVE ${ }^{\mathrm{TM}} 303$ cohort further suggested a relationship between BMI and weight gain; on average, patients with a higher BMI $\left(\mathrm{BMI}>30 \mathrm{~kg} / \mathrm{m}^{2}\right)$ treated with insulin detemir lost weight during the study, whilst patients at the lower end of the BMI spectrum $\left(<25 \mathrm{~kg} / \mathrm{m}^{2}\right)$ experienced modest weight gain (Meneghini et al 2007; Selam et al 2008). This observation is consistent with data from clinical trials (Hermansen et al 2006; Philis-Tsimikas et al 2006) and the PREDICTIVE ${ }^{\mathrm{TM}}$ study (Dornhorst et al 2007; Dornhorst et al 2008a, b).

Overall, PREDICTIVE ${ }^{\mathrm{TM}} 303$ successfully demonstrated patients' ability to adjust their basal insulin dose according to a simple dosing algorithm, and to be as effective in this respect as physician-directed dose adjustment. Both methods of adjustment were equally successful at reducing $\mathrm{HbA}_{1 \mathrm{c}}$ and FPG without increasing the risk of hypoglycemia, suggesting that a patient-driven method may be a safe and effective alternative to the physician-directed adjustment of insulin detemir.

\section{Insulin detemir delivery}

A reported $51 \%-79 \%$ of patients adhere to their insulin therapy; this percentage decreases as the complexity of 
a

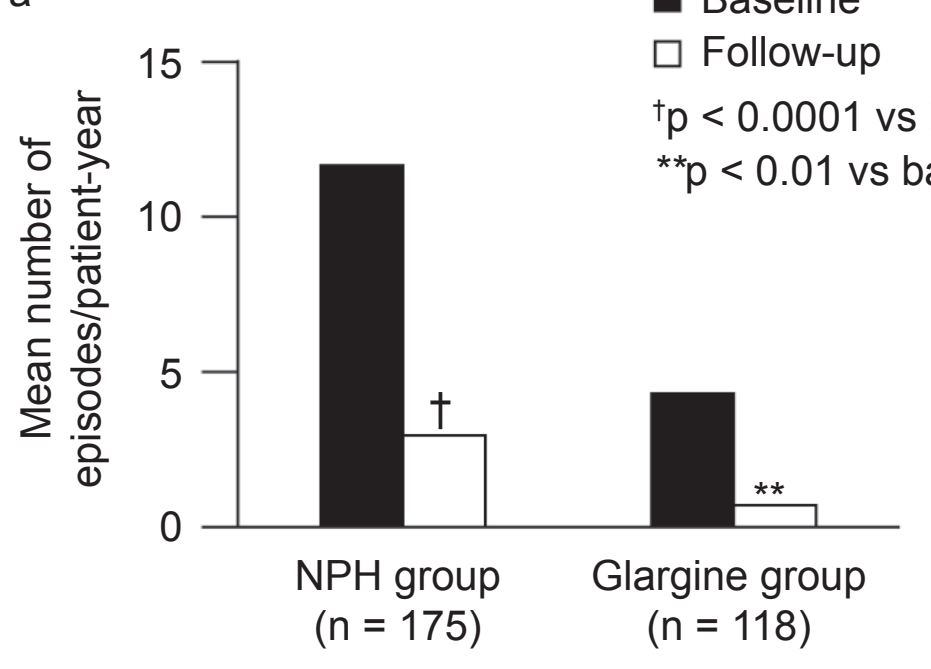

b

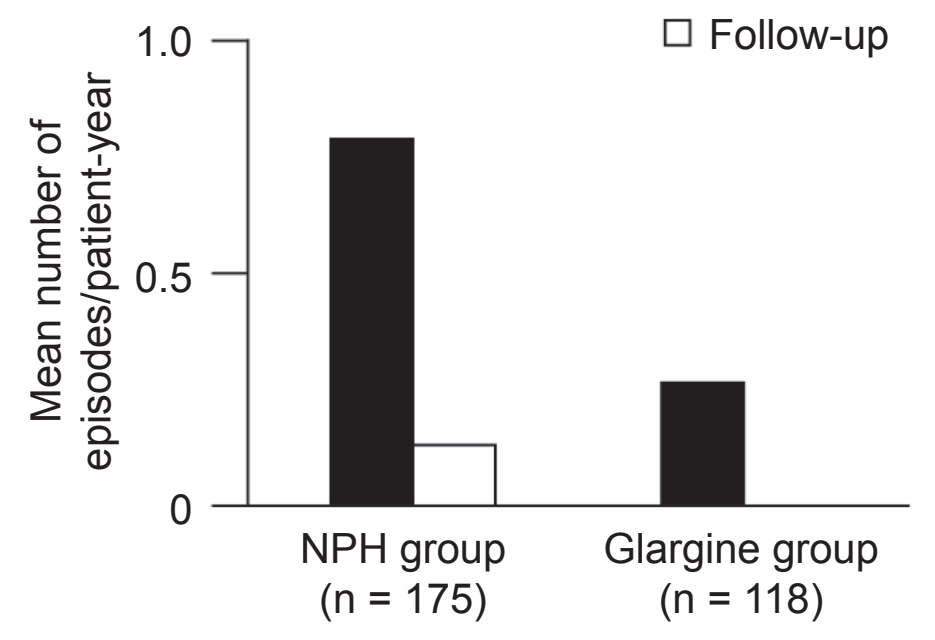

C

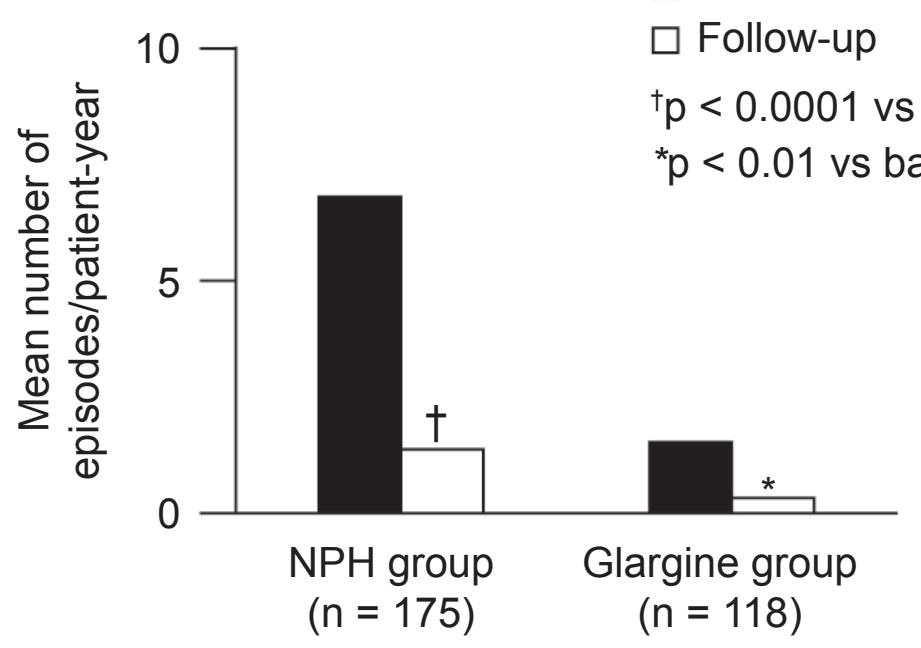

Figure 4 The number of hypoglycemic events (events/patient-year) in patients switching from NPH insulin or insulin glargine to treatment with insulin detemir. Reprinted with permission from Dornhorst A, Lüddeke HJ, Koenen C, et al 2008b. Transferring to insulin detemir from NPH insulin or insulin glarhine in type 2 diabetes patients on basal-only therapy with oral antidiabetic drugs improves glycemic control and reduces weight gain and risk of hypoglycemia: I4-week follow-up data from PREDICTIVE. Diabetes Obes Metab, 10:75-81. Copyright $\odot 2008$ Blackwell publishing. 
the regimen increases (Claxton et al 2001). Adherence to a prescribed therapeutic regimen is strongly influenced by the convenience and simplicity of the dosing regimen (Korytkowski 2002).

A common issue that affects patient adherence, particularly for new insulin users, is a reluctance to self-inject. Even confident patients would, naturally, prefer a painless and simple insulin delivery method. Insulin detemir is available for use with a vial and syringe, but also in two types of insulin delivery pen: NovoPen ${ }^{\circledR}$, a reusable insulin pen, and FlexPen $^{\circledR}$, a disposable pen. Both pens have been designed to facilitate greater patient acceptance, and a series of controlled trials have demonstrated that both devices improve patient satisfaction.

Modern basal insulins can only be effectively employed if dose delivery is accurate: achieving a reliable and correct dose of insulin is a key concern for both patients and physicians (Peyrot et al 2005). Up to $80 \%$ of people with diabetes incorrectly administer insulin when using a syringe (Korytkowski et al 2005). Previous studies have shown that patients using a vial and syringe are more likely to draw and inject an inaccurate dose, particularly when only small doses are required (Casella et al 1993; Lteif and Schwenk 1999).

Pen devices offer features which may minimize dosing inaccuracies during administration. For example, in a study by Korytkowski et al (2003), 85\% (89/105) of patients found it easier to read the insulin dose scale than with a conventional vial and syringe. Furthermore, $82 \%(86 / 105)$ of patients were reported more confident in setting the required dose, in contrast with only $11 \%(12 / 105)$ with the vial and syringe. As a result, $73 \%(77 / 105)$ of patients felt more confident with the accuracy of the insulin dose with a pen device compared with $19 \%$ (20/105) for vial/syringe (Korytkowski et al 2003).

Recent developments in insulin pens have proven popular with patients with regard to usability, convenience, perceptions of safety and trust and overall preference (Sommavilla et al 2008).

It is essential to maintain accuracy (within ISO recommendations) with the injection device as not to compromise glycemic control and lose patient confidence in their regimen.

\section{Summary}

When compared with older basal insulins, insulin detemir provides effective glycemic control, reduced variability in blood glucose-lowering response, less weight gain and a reduction in the number of hypoglycemic events, especially for nocturnal hypoglycemia. These results are supportive of the use of once-daily insulin detemir; dose adjustments of which have been made simpler by improvements in injection devices, and the development of simple, patient-friendly algorithms. Insulin detemir therefore provides a treatment option with the potential to lower the key barriers to acceptance of and adherence to insulin therapy in type 2 diabetes.

\section{Acknowledgments}

The author would like to thank Watermeadow Medical (Witney, UK) for their assistance in the preparation of this manuscript, which was supported by Novo Nordisk.

\section{Disclosures}

The author has no conflicts of interest to disclose.

\section{References}

Bock G, Wutte A, Köhler G, et al. 2008. Pharmacodynamics and pharmacokinetics of the long-acting insulin analogues detemir and glargine after 7 days of use and after their first administration in subjects with type 1 diabetes. [abstract] Diabetologia, 51(Suppl 1):S390.

Buchanan TA. 2003. Pancreatic beta-cell loss and preservation in type 2 diabetes. Clin Ther, 25(Supp1 B2):B32-B46.

Casella SJ, Mongilio MK, Plotnick LP, et al. 1993. Accuracy and precision of low-dose insulin administration. Pediatrics, 91(6):1155-7

Claxton AJ, Cramer J, Pierce C. 2001. A systematic review of the associations between dose regimens and medication compliance. Clin Ther, 23(8): $1296-310$

Davies M. 2004. The reality of glycaemic control in insulin treated diabetes: defining the clinical challenges. Int J Obes, 28(Suppl 2):S14-S22.

Dornhorst A, Lüddeke HJ, Koenen C, et al. 2008b. Transferring to insulin detemir from NPH insulin or insulin glarhine in type 2 diabetes patients on basal-only therapy with oral antidiabetic drugs improves glycaemic control and reduces weight gain and risk of hypoglycaemia: 14-week follow-up data from PREDICTIVE. Diabetes Obes Metab, 10:75-81.

Dornhorst A, Lüddeke HJ, Sreenan S, et al. 2007. Safety and efficacy of insulin detemir in clinical practice: 14-week follow-up data from type 1 and type 2 diabetes patients in the PREDICTIVE European cohort. Int J Clin Pract, 61:523-8.

Dornhorst A, Lüddeke H-J, Sreenan S, et al. on behalf of the PREDICTIVE study group. 2008a. Insulin detemir improves glycaemic control without weight gain in insulin-naive patients with type 2 diabetes: subgroup analysis from the PREDICTIVE ${ }^{\mathrm{TM}}$ study. Int J Clin Pract, 62(4):659-65.

Garber A, Calusen P, Pederson C, et al. 2007. Lower risk of hypoglycaemia with insulin detemir than with neutral protamine hagedorn insulin in older persons with type 2 diabetes: A pooled analysis of phase III trials. $J$ Am Geriatr Soc, 55:1735-40.

Gough SC. 2007. A review of human and analogue insulin trials. Diabetes Res Clin Pract, 77:1-15.

Haak T, Tiengo A, Draeger E, et al. 2005. Lower within-subject variability of fasting blood glucose and reduced weight gain with insulin detemir compared to NPH insulin in patients with type 2 diabetes. Diabetes Obes Metab, 7:56-64.

Havelund S, Plum A, Ribel U, et al. 2004. The mechanism of protraction of insulin detemir, a long-acting, acylated analogue of human insulin. Pharm Res, 21(8):1498-504.

Heise T, Nosek L, Ronn BB, et al. 2004. Lower within-subject variability of insulin detemir in comparison to NPH insulin and insulin glargine in people with type 1 diabetes. Diabetes, 53(6):1614-20.

Heise T, Pieber TR. 2007. Towards peakless, reproducible and long-acting insulins. An assessment of the basal analogues based on isoglycaemic clamp studies. Diabetes Obes Metab, 9:648-5. 
Heller S, Kozlovski P, Kurtzhals P. 2007. Insulin's 85th anniversary. An enduring medical miracle. Diabetes Res Clin Pract, 78:149-58.

Hermansen K, Davies M, Derezinski T, et al. 2006. A 26-week, randomized, parallel, treat-to-target trial comparing insulin detemir with NPH insulin as add-on therapy to oral glucose-lowering drugs in insulin-naive people with type 2 diabetes. Diabetes Care, 29:1269-74.

Hirsch IB, Brownlee M. 2007. The effect of glucose variability on the risk of microvascular complications in type 1 diabetes: response to author. Diabetes Care, 30(1):186-7.

Home P and Kurtzhals P. 2006. Insulin detemir: from concept to clinical experience. Expert Opin Pharmacoether, 7(3):325-43.

Home P, Boulton A, Jimenez J, et al. 2003. Issues relating to the early or earlier use of insulin in type 2 diabetes. Pract Diab Int, 20:61-73.

International Diabetes Federation (IDF). 2006. Diabetes Atlas, 3rd edition. p. $28-58$.

Kilpatrick ES, Rigby AS, Atkin SL. 2006. The effect of glucose variability on the risk of microvascular complications in type 1 diabetes. Diabetes Care, 29(7): 1486-90.

Klein O, Lygne J, Endahl L, et al. 2007. Albumin-bound basal insulin analogues (insulin detemir and NN344): comparable time-action profiles but less variability than insulin glargine in type 2 diabetes. Diabetes Obes Metab, 9(3):290-9.

Korytkowski M, Bell D, Jacobsen C, et al. for the Flexpen Study team. 2003. A multicenter, randomized, open-label, comparative, twoperiod crossover trial of preference, efficacy and safety profiles of a prefilled, disposable pen and conventional vial/syringe for insulin injection in patients with type 1 or type 2 diabetes mellitus. Clin Ther, 25(11):2836-48.

Korytkowski M, Niskanen L, Asakura T. 2005. Flexpen: addressing issues of confidence and convenience in delivery. Clin Ther, 27(Suppl 2): S89-100.

Korytkowski M. 2002. When oral agents fail: practical barriers to starting insulin. Int J Obes Relat Metab Disord, 26(Suppl 3):S18-24.

Kurtzhals P, Havelund S, Jonassen I, et al. 1997. Effect of fatty acids and selected drugs on the albumin binding of a long-acting, acylated insulin analogue. J Pharm Sci, 86(12):1365-8.

Kurtzhals P, Schaffer L, Sorensen A, et al. 2000. Correlations of receptor binding and metabolic and mitogenic potencies of insulin analogs designed for clinical use. Diabetes, 49(6):999-1005.

Kurtzhals P. 2004. Engineering predictability and protraction in a basal insulin analogue: the pharmacology of insulin detemir. Int $J$ Obes, 28(Suppl 2):S23-8

Lepore M, Pampanelli S, Fanelli C, et al. 2000. Pharmacokinetics and pharmacodynamics of subcutaneous injection of long-acting human insulin analog glargine, NPH insulin, and ultralente human insulin and continuous subcutaneous infusion of insulin lispro. Diabetes, 49:2142-8.

Lteif AN, Schwenk WF. 1999. Accuracy of pen injectors versus insulin syringes in children with type 1 diabetes. Diabetes Care, 22(1):137-40

Lüddeke HJ, Sreenan S, Aczel S, et al. PREDICTIVE Study Group. 2007. PREDICTIVE- a global, prospective observational study to evaluate insulin detemir treatment in types 1 and 2 diabetes: baseline characteristics and predictors of hypoglycaemia from the European cohort. Diabetes Obes Metab, 9(3):428-34.
Meneghini L, Koenen C, Weng W, et al. 2007. The usage of a simplified self-titration dosing guideline (303 Algorithm) for insulin detemir in patientswith type 2 diabetes - results of the randomized, controlled PREDICTIVE $^{\text {TM }} 303$ study. Diabetes Obes Metab, 9:902-13.

Monnier L, Colette C, Leiter L, et al. on behalf of the PGR group. 2007. The effect of glucose variability on the risk of microvascular complications in type 1 diabetes: response to author. Diabetes Care, 30(1):185-6.

Muggeo M, Zoppini G, Bonora E, et al. 2000. Fasting plasma glucose variability predicts 10 -year survival of type 2 diabetic patients: the Verona Diabetes Study. Diabetes Care, 23(1):45-50.

Nathan D, Buse J, Davisdson M, et al. 2006. Management of hyperglycaemia in type 2 diabetes: A consensus algorithm for the initiation and adjustment of therapy. Diabetes Care, 29:1963-72.

Niskanen L, Virkamäki A, Hansen JB, et al. 2008. Reducing fasting blood glucose variability is associated with a lower incidence of nocturnal hypoglycaemic events after starting insulin detemir: 3 month PREDICTIV ${ }^{\mathrm{TM}}$ data from 11 European countries. [abstract] Diabetologia, 51(Suppl 1):S388.

Peyrot M, Rubin RR, Lauritzen T, et al. on behalf of the International DAWN Advisory Panel. 2005. Resistance to insulin therapy among patients and providers results of the cross-national Diabetes Attitudes, Wishes, and Needs (DAWN) study. Diabetes Care, 28:2673-9.

Philis-Tsimikas A, Charpentier G, Clauson P, Martinez Ravn G, Roberts VL, Thorsteinsson B. 2006. Comparison of once-daily insulin detemir with NPH insulin added to a regimen of oral antidiabetic drugs in poorly controlled type 2 diabetes. Clin Ther, 28:1569-81.

Polonsky WH, Fisher L, Guzman S, Villa-Caballero L, Edelman SV. 2005. Psychological insulin resistance in patients with type 2 diabetes. Diabetes Care, 28:2543-5.

Rašlová K, Bogoev M, Raz I, et al. 2004. Insulin detemir and insulin aspart: a promising basal-bolus regimen for type 2 diabetes. Diabetes Res Clin Pract, 66(2):193-201.

Rosenstock J, Davies M, Home PD, et al. 2008. A randomised, 52-week, treat-to-target trial comparing insulin detemir with insulin glargine when administered as add-on to glucose-lowering drugs in insulin-naive people with type 2 diabetes. Diabetologia, 51(3):408-16.

Russell-Jones D, Khan R. 2007. Insulin-associated weight gain in diabetes - causes, effects and coping strategies. Diabetes Obes Metab, 9(6):799-812.

Selam JL, Koenen C, Weng W, et al. 2008. Improving glycemic control with insulin detemir using the 303 Algorithm in insulin naïve patients with type 2 diabetes: a subgroup analysis of the US PREDICTIVE 303 study. Curr Med Res Opin, 24(1):11-20.

Sommavilla B, Jørgensen C, Jensen KH. 2008. Safety, simplicity and convenience of a modified prefilled insulin pen. Expert Opin Pharmacother, 9(13):2223-32.

United Kingdom Prospective Diabetes Study; UKPDS 33. 1998. Intensive blood-glucose control with sulphonylureas or insulin compared with conventional treatment and risk of complications in patients with type 2 diabetes. Lancet, 352:837-53.

United Kingdom Prospective Diabetes Study; UKPDS 35. 2000. Association of glycaemia with macrovascular and microvascular complications of type 2 diabetes (UKPDS 35): prospective observational study. BMJ, 321:405-12.

WHO. World Health Organization. Accessed July 2008. URL: www. who.org. 